\title{
The Kirchhoff transformation and the Fick's second law with concentration-dependent diffusion coefficient
}

\author{
R. M. S. GAMA ${ }^{1}$, R. PAZETTO S. GAMA ${ }^{1,2}$ \\ ${ }^{1}$ Mechanical Engineering Department, Rio de Janeiro State University, 524 S. F. Xavier st \\ ${ }^{2}$ Department of Informatics, Pontifical Catholic University of Rio de Janeiro, 225 M. S. Vicente st \\ Rio de janeiro, BRAZIL
}

\begin{abstract}
In this work it is considered the Fick's second law in a context in which the diffusion coefficient depends on the concentration. It is employed the Kirchhoff transformation in order to simplify the mathematical structure of the Fick's second law, giving rise to a more convenient description. In order to provide a general protocol, the diffusion coefficient will be assumed a piecewise constant function of the concentration. Exact formulas are presented for both the Kirchhoff transformation and its inverse, in such a way that there is no limit of accuracy. Some numerical examples are presented with the aid of a semi-implicit procedure associated with a finite difference approximation.
\end{abstract}

Key-Words: Second Fick's Law, Concentration-Dependent Diffusion, Kirchhoff Transformation, Nonlinear Differential Equation, Semi Implicit Numerical Scheme, Finite Difference.

Received: January 26, 2021. Revised: May 30, 2021. Accepted: June 19, 2021. Published: July 8, 2021.

\section{Introduction}

The diffusion coefficient [1] can be assumed to be a constant in many situations. In particular, the constant diffusion coefficient hypothesis seems to be a good assumption when we study the diffusion of gases.

Nevertheless, when denser fluids are involved, the constant diffusion coefficient hypothesis may lie far from the reality $[2,3,4]$.

In particular, when the molecular interactions between the diffusing components are not negligible, the diffusion coefficient becomes dependent on the concentration, in a way that the relationship between them should be taken into account.

The main subject of this work is the Fick's second law $[5,6]$ in a context in which the diffusion coefficient $D$ is a function of the concentration $c$.

It is to be noticed that Fick's Second Law and its mathematical analysis is an issue each time more frequent in the current literature. Botar and Ruff [7] treats the diffusion as a random walk on a cubic lattice. Jou et al. [8] consider a higher order hydrodynamics for material motion in a medium under arbitrary nonequilibrium conditions. Van Milligen et al. [9] study the laws of Fick and of Fokker-Planck and present some numerical results. Sasaki et al. [10] investigate the confidence concerning Fick's Law in soils. In reference [11], authored by Zhang et al., it is considered the variation of the diffusion coefficient with the time.
Siepmann and Siepmann [12] present a study of the diffusion process applied to the drug delivery process. Guenneau and Puvirajesinghe [13] consider phenomena involving anisotropic diffusion.

In this paper we will restrict our attention to phenomena described by the following nonlinear parabolic partial differential equation [14]

$$
\frac{\partial c}{\partial t}=\frac{\partial}{\partial x}\left(D \frac{\partial c}{\partial x}\right)+\frac{\partial}{\partial y}\left(D \frac{\partial c}{\partial y}\right)+\frac{\partial}{\partial z}\left(D \frac{\partial c}{\partial z}\right)
$$

in which

$$
D=\hat{D}(c)>0
$$

The nonlinear partial differential equation represented by equation (1) is subjected to initial data and to boundary conditions [15].

The unknown is the concentration $c$, which may depend on the position $(x, y, z)$ and on the time.

\section{The Kirchhoff Transformation}

The Kirchhoff transformation is a largely employed tool for helping the description of heat transfer problems in which the thermal conductivity depends on the temperature [16-26].

Let us introduce the Kirchhoff transformation, within the mass transfer context, as follows

$$
\Phi=\hat{\Phi}(c)=\frac{1}{D_{0}} \int_{0}^{c} \hat{D}(\lambda) d \lambda, \quad D=\hat{D}(c)
$$

in which the constant $D_{0}$ could, for instance, represent the diffusion coefficient when the concentration is zero (but not necessarily). 
It is easy to see that

$$
\frac{\partial \Phi}{\partial x}=\frac{\partial}{\partial x} \frac{1}{D_{0}} \int_{0}^{c} \hat{D}(\lambda) d \lambda=\frac{D}{D_{0}} \frac{\partial c}{\partial x}
$$

and, consequently,

$$
\frac{\partial^{2} \Phi}{\partial x^{2}}=\frac{1}{D_{0}} \frac{\partial}{\partial x}\left(D \frac{\partial c}{\partial x}\right)
$$

The same holds for the derivatives with respect to $y$ and to $z$.

In addition,

$$
\frac{\partial \Phi}{\partial t}=\frac{\partial}{\partial t} \frac{1}{D_{0}} \int_{0}^{c} \hat{D}(\lambda) d \lambda=\frac{D}{D_{0}} \frac{\partial c}{\partial t}
$$

With the above definition, we have that

$$
\begin{aligned}
& \frac{\partial c}{\partial t}=\frac{D_{0}}{D} \frac{\partial \Phi}{\partial t} \\
& \frac{\partial}{\partial x}\left(D \frac{\partial c}{\partial x}\right)=D_{0} \frac{\partial^{2} \Phi}{\partial x^{2}} \\
& \frac{\partial}{\partial y}\left(D \frac{\partial c}{\partial y}\right)=D_{0} \frac{\partial^{2} \Phi}{\partial y^{2}} \\
& \frac{\partial}{\partial z}\left(D \frac{\partial c}{\partial z}\right)=D_{0} \frac{\partial^{2} \Phi}{\partial z^{2}}
\end{aligned}
$$

and, so, equation (1) may be rewritten as

$$
\frac{1}{D} \frac{\partial \Phi}{\partial t}=\frac{\partial^{2} \Phi}{\partial x^{2}}+\frac{\partial^{2} \Phi}{\partial y^{2}}+\frac{\partial^{2} \Phi}{\partial z^{2}}
$$

in which the diffusion coefficient is, now, regarded as a function of $\Phi$. The functional relationship between the diffusion coefficient and the function $\Phi$ is ensured, provided the diffusion coefficient is always greater than a given positive constant. In other words, if there exists a constant $\varepsilon>0$ such that, for any concentration,

$$
D=\hat{D}(c) \geq \varepsilon>0
$$

One of the challenges in this work is to present an user friendly relationship between the diffusion coefficient and the concentration, providing an easy way for reaching the concentration $c$ once the function $\Phi$ is known.

Now, with the use of the Kirchhoff transformation, the only nonlinearity will be the one due to the diffusivity appearing in the transient term. It is to be noticed that the Kirchhoff transformation works well with discontinuous functions.

This fact induces us to consider piecewise constant relationships between the diffusion coefficient and the concentration.

\section{Piecewise Constant Approximation}

The relationship between the diffusion coefficient and the concentration is established from discrete experimental data. So it is always reasonable to consider the diffusion coefficient as a piecewise constant function of the concentration. This is illustrated in figures 1,2 and 3.

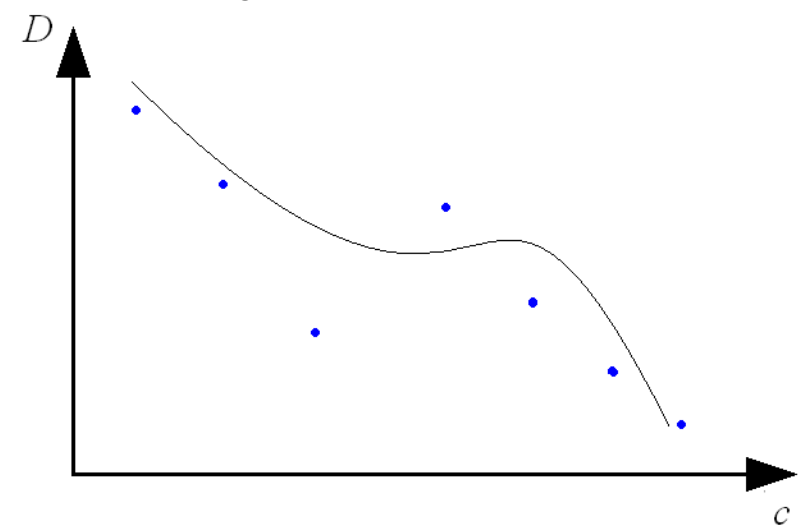

Fig. 1 - A typical curve fitted for seven given values.

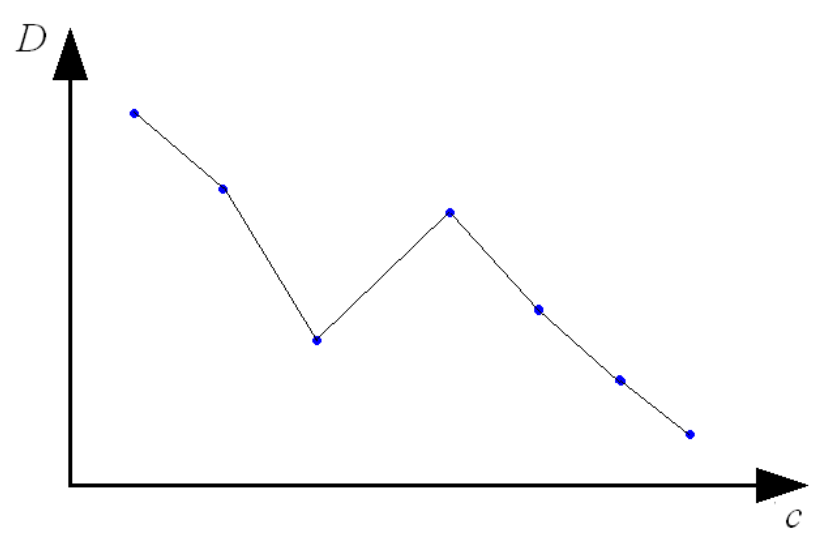

Fig. 2 - A typical piecewise linear approximation for the same values given in figure 1 .

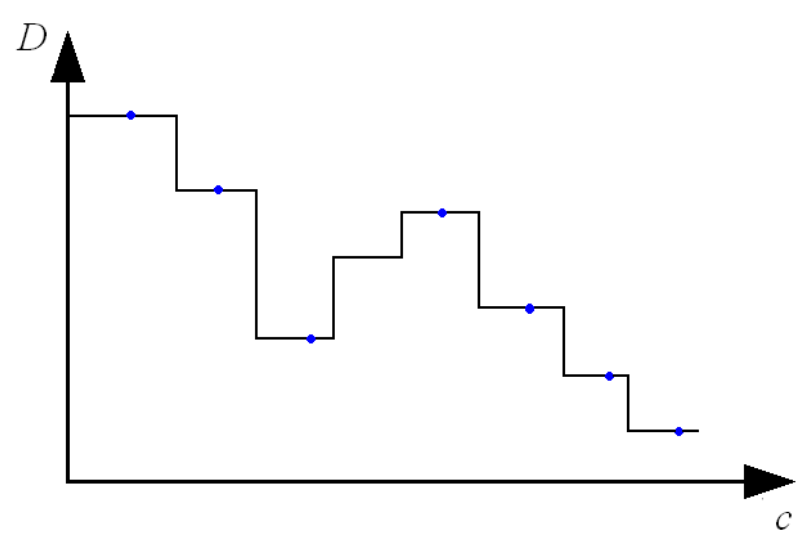

Fig. 3 - Illustration of a possible piecewise constant approximation for the same values given in figure 1 .

All approximations illustrated in the previous figures are valid, but not necessarily equivalent, since, when building a curve from sparse points, (for instance by means of the least squares method), it is possible to find functions for which the inversion of the Kirchhoff transformation is very complicated. 
The same can be said for the piecewise linear continuous approximation, whose inversion is usually complicated.

The piecewise constant approximation, on the other hand, has two undeniable advantages:

1- it always allows closed-form analytical inversion (as it will be shown later by equations (12) and (13)) 2- there is no precision limit, as long as there are enough points to be employed. In other words, we may use all the available data in an exact way. This becomes clear in equation (10).

These facts were fundamental for choosing to represent the diffusion coefficient $D$ as a piecewise constant function of the concentration $\mathrm{c}$. by

The piecewise constant approximation is given

$$
D=\hat{D}(c)=\left\{\begin{array}{c}
D_{1} \text { for } \mathrm{c} \leq c_{1} \\
D_{i} \quad \text { for } \mathrm{c}_{i} \geq c>c_{i-1}, \quad 1<i<N \\
D_{N} \text { para } \mathrm{c}>c_{N-1}
\end{array}\right.
$$

in which $D_{1}, D_{2}, \ldots, D_{N}$ and $c_{1}, c_{2}, \ldots, c_{N-1}$ are positive constants

Assuming that the diffusion coefficient is given by (10), the Kirchhoff transformation is given by

$$
\Phi=\left\{\begin{array}{c}
\frac{D_{1}}{D_{0}} c, \quad \text { if } \mathrm{c} \leq c_{1} \\
\frac{1}{D_{0}}\left\{D_{2}\left(c-c_{1}\right)+D_{1} c_{1}\right\}, \quad \text { if } \mathrm{c}_{2} \geq c>c_{1} \\
-------------- \\
\frac{1}{D_{0}}\left\{D_{N}\left(c-c_{N-1}\right)+D_{N-1}\left(c_{N-1}-c_{N-2}\right)+\right. \\
\left.\ldots+D_{2}\left(c_{2}-c_{1}\right)+D_{1} c_{1}\right\}, \quad \text { if } \mathrm{c}>c_{N-1}
\end{array}\right.
$$

The above relation yields

$$
\begin{aligned}
& \Phi=\frac{1}{2 D_{0}}\left(D_{1}+D_{N}\right) c+ \\
& +\frac{1}{2 D_{0}}\left(\sum_{i=2}^{N}\left(D_{i}-D_{i-1}\right)\left(\left|c-c_{i-1}\right|-c_{i-1}\right)\right)
\end{aligned}
$$

Defining the (nonnegative) constants $\Phi_{1}, \Phi_{2}$, $\Phi_{3}, \ldots, \Phi_{N-1}$ as follows

$$
\Phi_{i}=\sum_{j=1}^{i} \frac{D_{j}}{D_{0}}\left(c_{j}-c_{j-1}\right), \quad 1 \leq i \leq N-1, \mathrm{c}_{0}=0
$$

the inverse of the Kirchhoff transformation is unique and explicitly given by

$$
\begin{aligned}
c=\frac{D_{0}}{2} & \left\{\left(\frac{1}{D_{1}}+\frac{1}{D_{N}}\right) \Phi+\right. \\
& \left.+\sum_{i=2}^{N}\left(\frac{1}{D_{i}}-\frac{1}{D_{i-1}}\right)\left(\left|\Phi-\Phi_{i-1}\right|-\Phi_{i-1}\right)\right\}
\end{aligned}
$$

The above relationship holds for any piecewise constant approximation and, thus, it is completely general.

For instance, when $N=2$, we have [18]

$$
D=\hat{D}(c)=\left\{\begin{array}{lll}
D_{1} & \text { for } & \mathrm{c} \leq c_{1} \\
D_{2} & \text { for } & \mathrm{c}>c_{1}
\end{array}\right.
$$

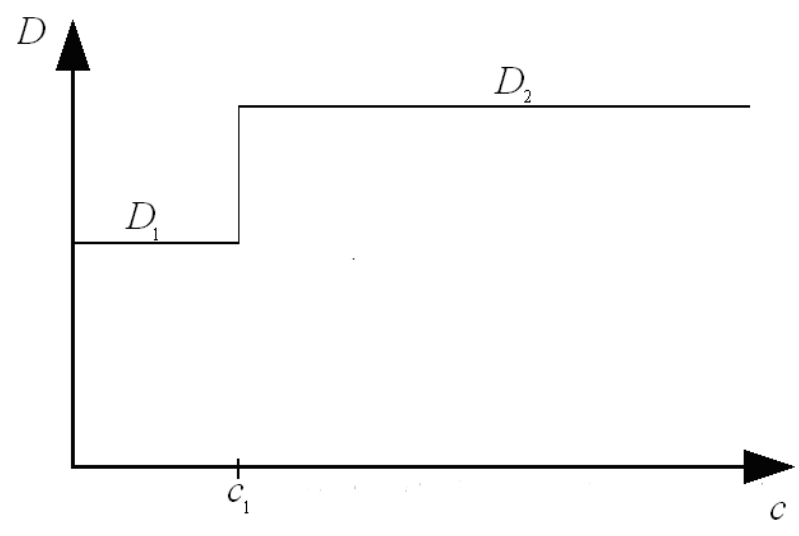

Fig. 4 - An approximation with two steps $(N=2)$.

In such case we have

$\Phi=\frac{1}{2}\left\{\left(\frac{D_{1}}{D_{0}}+\frac{D_{2}}{D_{0}}\right) c+\left(\frac{D_{2}}{D_{0}}-\frac{D_{1}}{D_{0}}\right)\left(\left|c-c_{1}\right|-c_{1}\right)\right\}($

while the inverse is given by

$$
\begin{aligned}
c= & \frac{1}{2}\left\{\left(\frac{D_{0}}{D_{1}}+\frac{D_{0}}{D_{2}}\right) \Phi+\right. \\
& \left.+\left(\frac{D_{0}}{D_{2}}-\frac{D_{0}}{D_{1}}\right)\left(\left|\Phi-\frac{D_{1}}{D_{0}} c_{1}\right|-\frac{D_{1}}{D_{0}} c_{1}\right)\right\}
\end{aligned}
$$

For instance, when $N=10$, we have

$$
D=\hat{D}(c)=\left\{\begin{array}{c}
D_{1} \text { for } \quad \mathrm{c} \leq c_{1} \\
D_{2} \text { for } \quad \mathrm{c}_{2} \geq c>c_{1} \\
D_{3} \text { for } \quad \mathrm{c}_{3} \geq c>c_{2} \\
D_{4} \text { for } \quad \mathrm{c}_{4} \geq c>c_{3} \\
D_{5} \text { for } \quad c_{5} \geq c>c_{4} \\
D_{6} \text { for } c_{6} \geq c>c_{5} \\
D_{7} \text { for } \quad c_{7} \geq c>c_{6} \\
D_{8} \text { for } \quad c_{8} \geq c>c_{7} \\
D_{9} \text { for } \quad c_{9} \geq c>c_{8} \\
D_{10} \text { for } \quad c>c_{9}
\end{array}\right.
$$

and the following associated figure. 


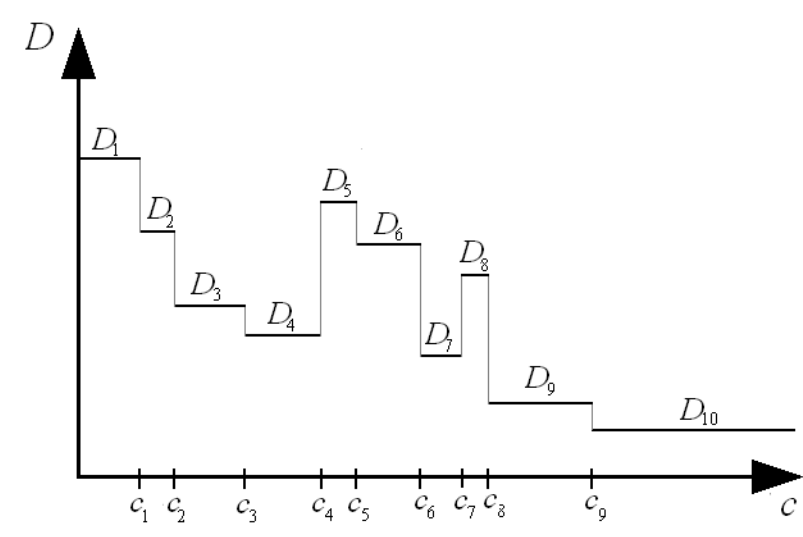

Fig. 5 - An approximation with ten steps $(N=10)$.

\section{An Error Estimate}

Let us assume that the diffusion coefficient is known within the range $c_{M I N} \leq c \leq c_{M A X}$ and given by means of the function $D=\hat{D}(c)$ such that

$$
D=\hat{D}(c), \quad c_{\text {MIN }} \leq c \leq c_{\text {MAX }}
$$

Let us consider a piecewise constant approximation in which $c_{1}=c_{M I N}, c_{N-1}=c_{M A X}$, $D_{1}=\hat{D}\left(c_{M I N}\right), D_{N}=\hat{D}\left(c_{M A X}\right)$ and $c_{i+1}-c_{i}=\Delta c_{i+1}$, as suggested in figure 6 .
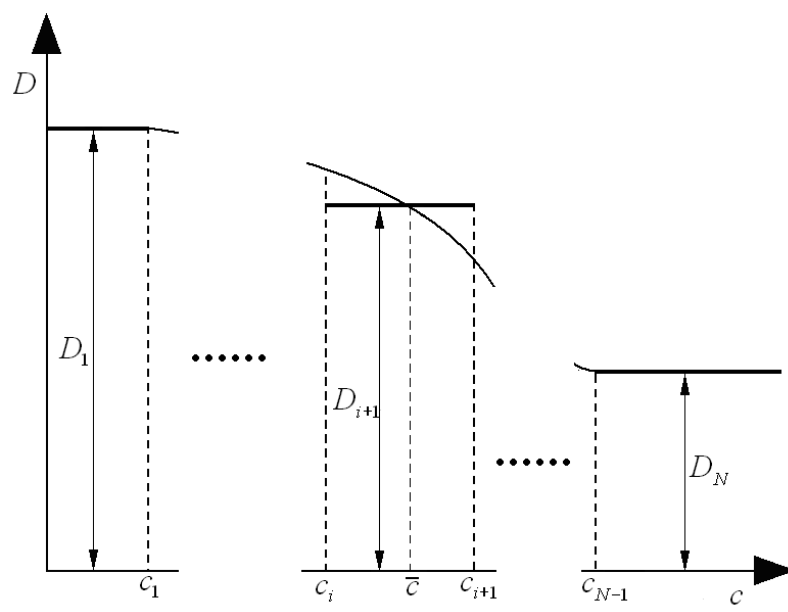

Fig. 6-A scheme for helping the error estimate.

So, the exact Kirchhoff transformation, denoted here by $\Phi_{\text {EXACT }}$, is the area below the curve (called exact), between zero and $c$, divided by $D_{0}$, given by

$$
\begin{array}{r}
\Phi_{E X A C T}=\frac{1}{D_{0}} \int_{0}^{c} \hat{D}(\lambda) d \lambda=\frac{1}{D_{0}} \int_{0}^{c_{1}} \hat{D}(\lambda) d \lambda+ \\
+\frac{1}{D_{0}} \int_{c_{1}}^{c_{2}} \hat{D}(\lambda) d \lambda+\ldots+\frac{1}{D_{0}} \int_{c_{j-1}}^{c} \hat{D}(\lambda) d \lambda
\end{array}
$$

The approximate Kirchhoff transformation, obtained with the piecewise constant approximation, is the area of the rectangles between zero and $c$ (divided by $D_{0}$ ), given by

$$
\Phi_{\text {APPROX }}=\frac{D_{1}}{D_{0}} c_{1}+\frac{D_{2}}{D_{0}}\left(c_{2}-c_{1}\right)+\ldots+\frac{D_{j}}{D_{0}}\left(c-c_{j-1}\right)
$$

Choosing the constants $D_{j}$ as (mean value)

$$
D_{i}=\frac{1}{c_{i}-c_{i-1}} \int_{c_{i-1}}^{c_{i}} \hat{D}(\lambda) d \lambda=\frac{1}{\Delta c} \int_{c_{i-1}}^{c_{i}} \hat{D}(\lambda) d \lambda
$$

the difference between the exact and the approximate value of the Kirchhoff transformation is obtained from

$$
\begin{aligned}
& \Phi_{\text {EXACT }}-\Phi_{\text {APPROX }}= \\
& =\frac{1}{D_{0}}\left\{\int_{c_{i-1}}^{c} \hat{D}(\lambda) d \lambda-\left(\frac{c-c_{i-1}}{c_{i}-c_{i-1}}\right) \int_{c_{i-1}}^{c_{i}} \hat{D}(\lambda) d \lambda\right\}
\end{aligned}
$$

Hence, for $c=c_{i-1}$ and for $c=c_{i}$ the difference is zero. Therefore, the maximum difference will be reached when the derivative of $\Phi_{\text {EXACT }}-\Phi_{A P P R O X}$ with respect to $c$ is zero, within the open interval $\left(c_{i-1}, c_{i}\right)$. This derivative is given by

$$
\begin{aligned}
& \frac{d}{d c}\left\{\Phi_{\text {EXACT }}-\Phi_{\text {APPROX }}\right\}= \\
& =\frac{1}{D_{0}}\left\{\hat{D}(c)-\frac{1}{\Delta c} \int_{c_{i-1}}^{c_{i}} \hat{D}(\lambda) d \lambda\right\}=\frac{\hat{D}(c)-D_{i}}{D_{0}}
\end{aligned}
$$

and, so, the maximum difference (for this interval) occurs for $c=\bar{c}$ such that

$$
\hat{D}(\bar{c})=D_{i}
$$

Defining the error as follows

$$
E R R O R=\left|\Phi_{\text {EXACT }}-\Phi_{\text {APPROX }}\right|
$$

the maximum error is reached when $c=\bar{c}$ at a given interval $\left(c_{j-1}, c_{j}\right)$. In other words,

$$
\operatorname{ERROR}_{M A X} \leq \max _{\substack{c_{j \rightarrow 1} \leq \lambda \leq c_{j} \\ 1<j<N}}\left|\frac{\hat{D}(\lambda)-D_{j}}{D_{0}}\right| \Delta c_{j}
$$

Since the difference between the maximum and the minimum diffusion coefficient is always bounded, the error will decrease as the step width decreases. This fact enables us to increase, as desired, the degree of accuracy.

The computational implementation of the formulas is very fast and easy and, therefore, $N$ can be increased as much as required in order to minimize the error. 


\section{Numerical Scheme}

In order to present some results, we shall consider a semi-implicit algorithm.

The use of the Kirchhoff transformation enables us to advance from time $t^{n}$ to time $t^{n+1}$ considering the term $\frac{\partial^{2} \Phi}{\partial x^{2}}+\frac{\partial^{2} \Phi}{\partial y^{2}}+\frac{\partial^{2} \Phi}{\partial z^{2}}$ evaluated at the time $t^{n+1}$. The only quantity evaluated at the time $t^{n}$ will be the diffusivity coefficient $D$.

This semi-implicit algorithm for advancing from $t^{n}$ to $t^{n+1}$ can be summarized as follows

$$
\frac{\Phi^{n+1}-\Phi^{n}}{D^{n} \Delta t}=\left[\frac{\partial^{2} \Phi}{\partial x^{2}}+\frac{\partial^{2} \Phi}{\partial y^{2}}+\frac{\partial^{2} \Phi}{\partial z^{2}}\right]^{n+1}
$$

in which the superscript $n$ indicates that the quantity is evaluated at the time $t^{n}$.

In the above equation $D^{n}$ depends on $\Phi^{n}$. So, the approximation at $t^{n+1}$ is obtained from a linear problem, especially when we have Dirichlet and/or Neumann boundary conditions.

If we employ a centered finite difference approximation, equation (28) becomes

$$
\begin{gathered}
\frac{\Phi_{i, j, k}^{n+1}-\Phi_{i, j, k}^{n}}{D^{n} \Delta t}=\frac{\Phi_{i-1, j, k}^{n+1}-2 \Phi_{i, j, k}^{n+1}+\Phi_{i+1, j, k}^{n+1}}{(\Delta x)^{2}}+ \\
+\frac{\Phi_{i, j-1, k}^{n+1}-2 \Phi_{i, j, k}^{n+1}+\Phi_{i, j+1, k}^{n+1}}{(\Delta y)^{2}}+ \\
+\frac{\Phi_{i, j, k-1}^{n+1}-2 \Phi_{i, j, k}^{n+1}+\Phi_{i, j, k+1}^{n+1}}{(\Delta z)^{2}}
\end{gathered}
$$

in which $\Phi_{i, j, k}^{n}$ represents the approximation for $\Phi$ at the spatial point $\left(x_{i}, y_{j}, z_{k}\right)$ at the time $t^{n}$. The spatial coordinates are given by

$x_{i}=(i-1) \Delta x ; \quad y_{j}=(j-1) \Delta y ; \quad z_{k}=(k-1) \Delta z$

and the time $t^{n}$ is given by

$$
t^{n}=n \Delta t
$$

Equation (29) holds for spatial points $\left(x_{i}, y_{j}, z_{k}\right)$ that do not belong to the boundary.

Equation (27) represents a semi-implicit scheme since the diffusion coefficient is evaluated at the time $t^{n}$ and not at the time $t^{n+1}$.

Once known $\Phi_{i, j, k}^{n}$, the concentration $c_{i, j, k}^{n}$ is obtained from the inverse of the Kirchhoff transformation. Clearly, $c_{i, j, k}^{n}$ represents the concentration at the spatial point $\left(x_{i}, y_{j}, z_{k}\right)$ at the time $t^{n}$.

\section{Some One Dimensional Results}

In order to illustrate the proposed procedure and the effect of the concentration dependent diffusivity coefficient, let us consider the following one dimensional problem (with Dirichlet boundary conditions [14])

$$
\begin{aligned}
& \frac{\partial c}{\partial t}=\frac{\partial}{\partial x}\left(D \frac{\partial c}{\partial x}\right), \quad x \in(0, L), \quad t>0 \\
& D=\hat{D}(c)=\left\{\begin{array}{l}
D_{1}=\text { constant }, \quad c \leq 1 / 2 \\
D_{2}=\text { constant }, \quad 1 / 2<c
\end{array}\right. \\
& c=C_{A} \quad \text { at } \quad x=0, \quad t>0 \\
& c=C_{B} \quad \text { at } \quad x=L, \quad t>0 \\
& c=0 \text { for } \quad x \in(0, L), \quad t=0
\end{aligned}
$$

With the aid of the Kirchhoff transformation, poblem (32) becomes

$$
\begin{aligned}
& \frac{1}{D} \frac{\partial \Phi}{\partial t}=\frac{\partial^{2} \Phi}{\partial x^{2}}, \quad x \in(0, L), \quad t>0 \\
& D=\tilde{D}(\Phi)=\left\{\begin{array}{l}
D_{1}=\text { constant }, \quad \Phi \leq \Phi_{1} \\
D_{2}=\text { constant }, \quad \Phi_{1}<\Phi
\end{array}\right. \\
& \Phi=\Phi_{A} \quad \text { at } \quad x=0, \quad t>0 \\
& \Phi=\Phi_{B} \quad \text { at } \quad x=L, \quad t>0 \\
& \Phi=0 \text { for } \quad x \in(0, L), \quad t=0
\end{aligned}
$$

in which, from equation (16) we have

$$
\begin{aligned}
& \Phi_{1}=c_{1} \frac{D_{1}}{D_{0}} \\
& \Phi_{A}=\left(\frac{D_{1}+D_{2}}{2 D_{0}}\right) C_{A}+\left(\frac{D_{1}-D_{2}}{2 D_{0}}\right)\left(\left|C_{A}-\frac{1}{2}\right|-\frac{1}{2}\right) \\
& \Phi_{B}=\left(\frac{D_{1}+D_{2}}{2 D_{0}}\right) C_{B}+\left(\frac{D_{1}-D_{2}}{2 D_{0}}\right)\left(\left|C_{B}-\frac{1}{2}\right|-\frac{1}{2}\right)
\end{aligned}
$$

In this case, the numerical scheme may be represented as follows (it is remarkable that $\bar{N}$ and $N$ have different meanings)

$$
\begin{aligned}
& \frac{\Phi_{i}^{n+1}-\Phi_{i}^{n}}{D^{n} \Delta t}=\frac{\Phi_{i+1}^{n+1}-2 \Phi_{i}^{n+1}+\Phi_{i-1}^{n+1}}{(\Delta x)^{2}}, \quad 1<i<\bar{N} \\
& \Phi_{1}^{n+1}=\Phi_{A} \\
& \Phi_{\bar{N}}^{n+1}=\Phi_{B} \\
& \Phi_{i}^{0}=0, \quad 1<i<\bar{N}
\end{aligned}
$$

in which

$$
\begin{array}{ll}
x_{i}=(i-1) \Delta x, & \Delta x=\frac{L}{\bar{N}-1} \\
t^{n+1}=t^{n}+\Delta t, & t^{0}=0
\end{array}
$$

Equation (35) represents, for each $n \geq 0$, a 
linear system whose unknowns are the $\Phi_{i}^{n+1}$ $(i=2,3,4, \ldots, \bar{N}-1)$. This system may be conveniently rewritten as

$$
\begin{aligned}
& \Phi_{i}^{n+1}=\frac{\left(\Phi_{i+1}^{n+1}+\Phi_{i-1}^{n+1}\right) D^{n} \Delta t+\Phi_{i}^{n}(\Delta x)^{2}}{(\Delta x)^{2}+2 D^{n} \Delta t}, 1<i<\bar{N} \\
& \Phi_{1}^{n+1}=\Phi_{A} \\
& \Phi_{\bar{N}}^{n+1}=\Phi_{B} \\
& \Phi_{i}^{0}=0, \quad 1<i<\bar{N}
\end{aligned}
$$

Considering a Neumann boundary condition [14] at $x=L$, we have, instead of (32), the following problem

$$
\begin{aligned}
& \frac{\partial c}{\partial t}=\frac{\partial}{\partial x}\left(D \frac{\partial c}{\partial x}\right), \quad x \in(0, L), \quad t>0 \\
& D=\hat{D}(c)=\left\{\begin{array}{l}
D_{1}=\text { constant }, \quad c \leq 1 / 2 \\
D_{2}=\text { constant }, \quad 1 / 2<c
\end{array}\right. \\
& c=C_{A} \quad \text { at } \quad x=0, \quad t>0 \\
& \frac{\partial c}{\partial x}=0 \quad \text { at } \quad x=L, \quad t>0 \\
& c=0 \quad \text { for } \quad x \in(0, L), \quad t=0
\end{aligned}
$$

In this case, the numerical scheme may be represented as follows

$$
\begin{aligned}
& \Phi_{i}^{n+1}=\frac{\left(\Phi_{i+1}^{n+1}+\Phi_{i-1}^{n+1}\right) D^{n} \Delta t+\Phi_{i}^{n}(\Delta x)^{2}}{(\Delta x)^{2}+2 D^{n} \Delta t}, 1<i<\bar{N} \\
& \Phi_{1}^{n+1}=\Phi_{A} \\
& \Phi_{\bar{N}}^{n+1}=\Phi_{\bar{N}-1}^{n+1} \\
& \Phi_{i}^{0}=0, \quad 1<i \leq \bar{N}
\end{aligned}
$$

in which the unknowns are the $\Phi_{i}^{n+1}$.

Figures 7 and 8 present some results for problem (33), assuming that $D_{1}=5 D_{2}$ and $c_{1}=0.5$.

In these figures we have a direct comparison between the constant diffusion coefficient approach (in which the diffusion coefficient is given by a mean value) and the piecewise constant diffusion coefficient, considering $N=2$.

In figure 7 we have the same boundary condition at $x=0$ and at $x=L \quad\left(C_{A}=C_{B}=1\right)$ while, in figure 8 , the boundary condition at $x=0$ and at $x=L$ are different $\left(C_{A}=1, C_{B}=0\right)$.

In both the figures it is easy to observe the jump on the first derivative of the concentration when $c=0.5$. This fact does not occurs under the constant diffusion coefficient hypothesis.

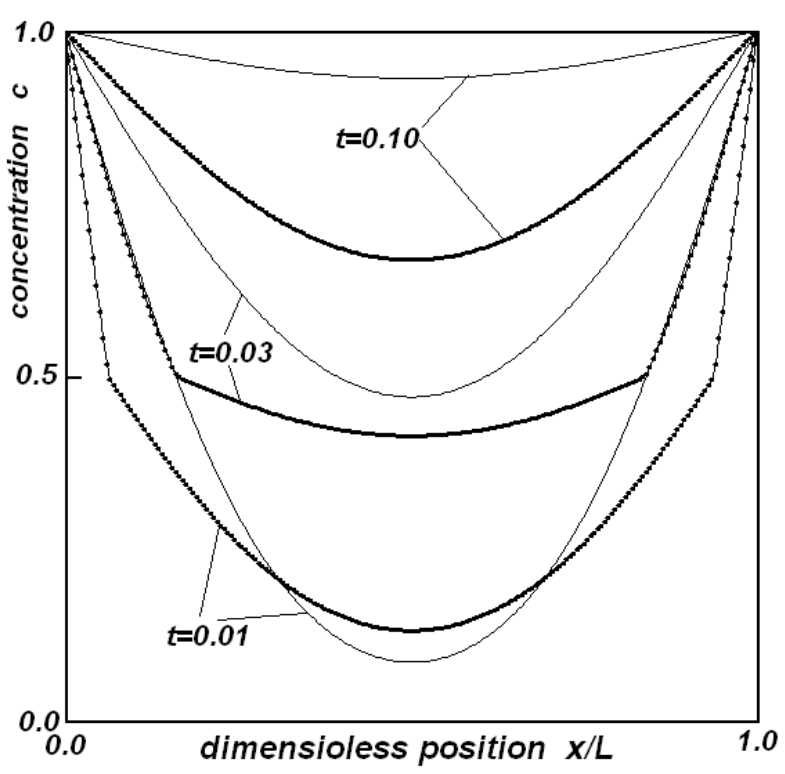

Fig. 7 - The concentration $c$ as a function of the dimensionless position $x / L$ at three time instants, assuming $C_{A}=C_{B}=1$ and $D_{1}=5 D_{2}$.

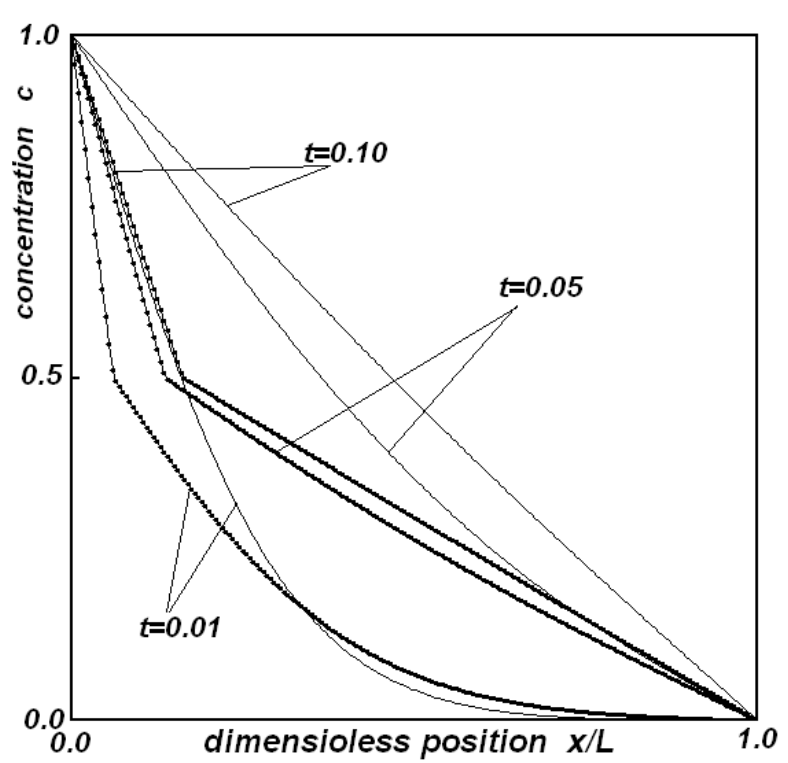

Fig. 8 - The concentration $c$ as a function of the dimensionless position $x / L$ at three time instants, assuming $C_{A}=1, C_{B}=0$ and $D_{1}=5 D_{2}$.

Figure 9 presents some results for problem (36), assuming that $D_{1}=5 D_{2}$ and $c_{1}=0.5$. As in the cases of figures 7 and 8 , we have a direct comparison between the constant diffusion coefficient approach and the piecewise constant diffusion coefficient, considering $N=2$. 


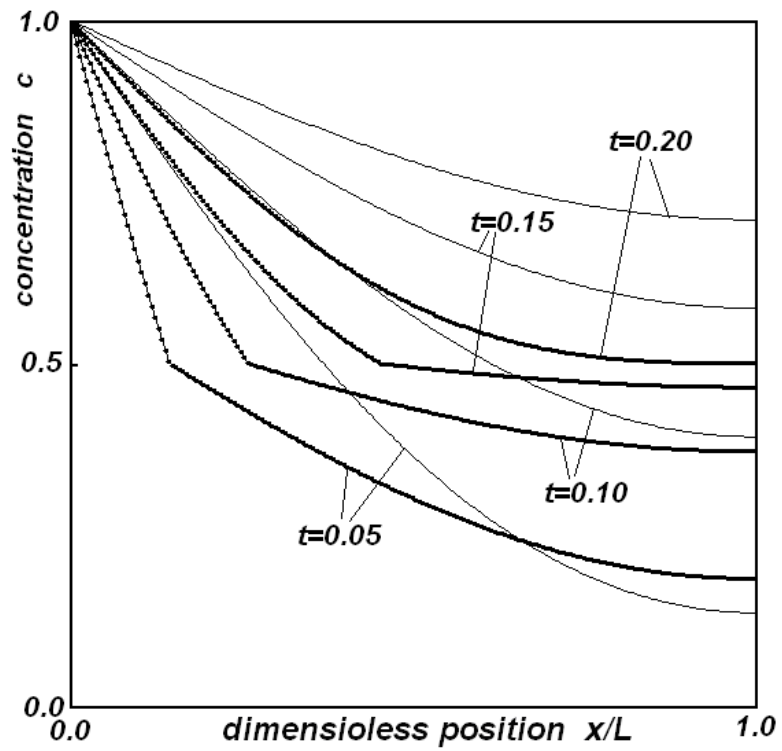

Fig. 9 - The concentration $c$ as a function of the dimensionless position $x / L$ at four time instants, assuming $C_{A}=1, \partial c / \partial x=0$ at $x=L$ and $D_{1}=5 D_{2}$.

In figures 7, 8 and 9 the thin line represents the result obtained under the constant diffusion coefficient assumption ( $D=3 D_{2}=$ constant). The employed number of nodes was $\bar{N}=201$.

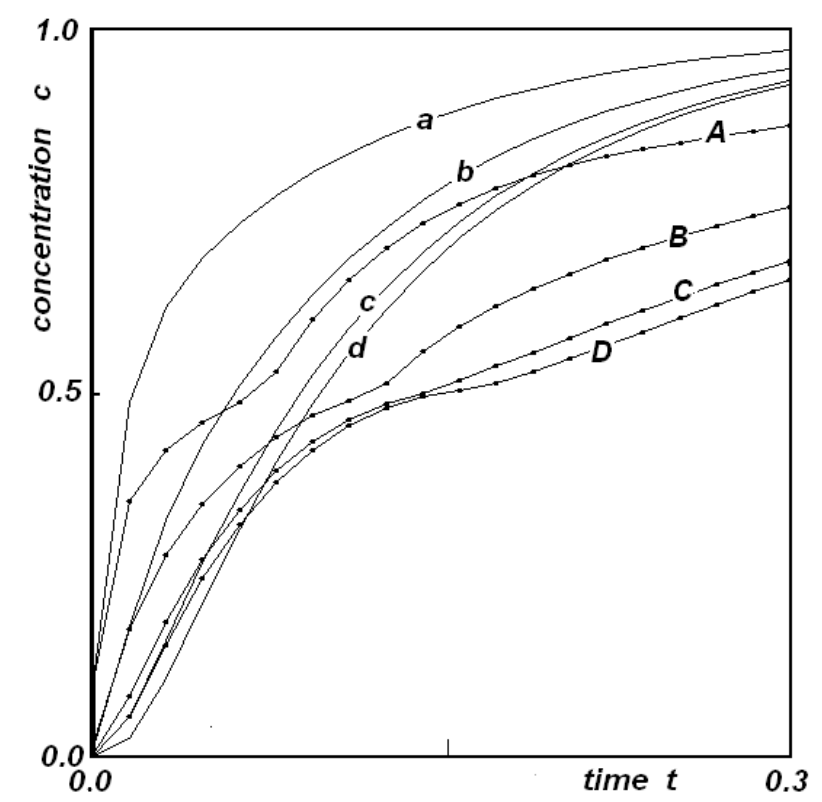

Fig. 10 - The concentration $c$ as a function of the time $t$, assuming $C_{A}=C_{B}=1$ and $D_{1}=5 D_{2}$, for $x / L=0.875$ and 0.125 (A and $\mathrm{a}$ ); 0.75 and 0.25 (B and b); 0.625 and 0.375 (C and c) and 0.5 (D and $d)$.

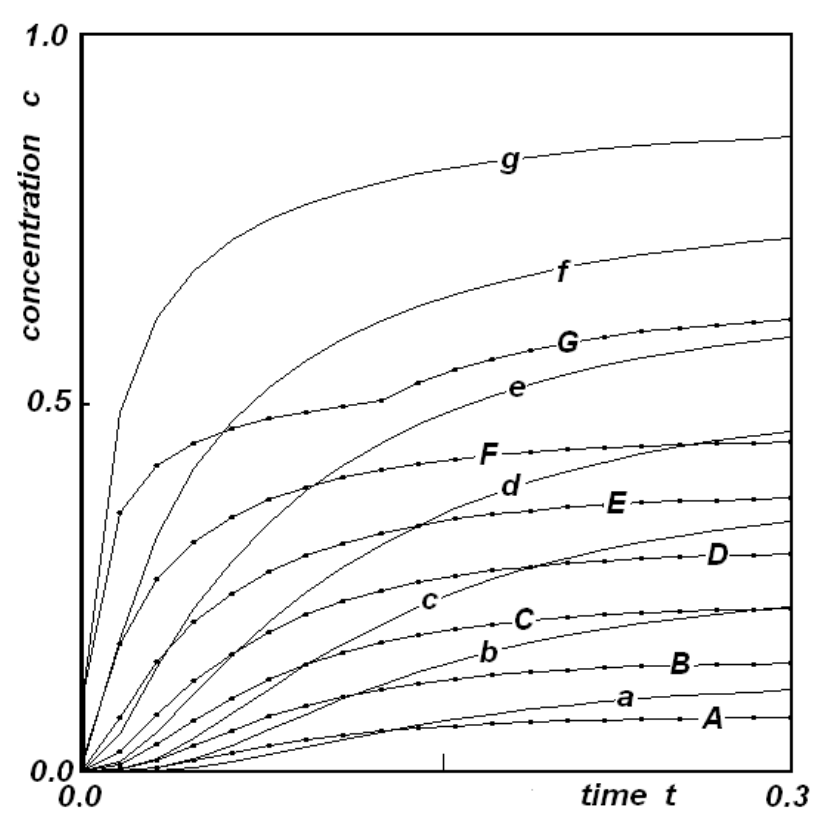

Fig. 11 - The concentration $c$ as a function of the time $t$, assuming $C_{A}=1, C_{B}=0$ and $D_{1}=5 D_{2}$, or $x / L=0.875$ (A and a); 0.75 (B and b); 0.625 (C and c); 0.5 (D and d); 0.375 (E and e); 0.25 (F and $\mathrm{f}$ ) and 0.125 ( $\mathrm{G}$ and $\mathrm{g}$ ).

In figures 10 and 11 it is presented the concentration as a function of time, for seven selected spatial points, for problem (32). In figure 10 we have $C_{A}=C_{B}=1$ (like in figure 7) while in figure 11 we have $C_{A}=1$ and $C_{B}=0$, like in figure 8.

The capital letters A, B, C, D, E, F and G indicates the results obtained with concentration-dependent diffusion coefficient, while the lower case letters a, b, c, d, e, f and $g$ indicates the results obtained under the constant diffusion coefficient assumption ( $D=3 D_{2}$ ).

The solution of systems (37) and (39) was carried out with the aid of a Gauss-Seidel scheme [15], by means of a very simple FORTRAN code. The figures were constructed with the aid of a specific JAVA code, created by the authors.

\section{Conclusions}

This work presented a powerful tool for describing and simulating the Fick's second law taking into account any relationship between the diffusion coefficient and the concentration.

Besides allowing the representation of any functional dependence between the diffusion coefficient and the concentration, the use of piecewise constant approximation gives rise to very 
simple closed form formulas for both the Kirchhoff transformations as well as its inverse, by means of convenient equations. In addition, there is no limit of accuracy for these approximations.

The computational implementation of the piecewise constant approximation becomes more interesting when it is taken into account that it does not require the use of any sophisticated function. It is needed only the basic math operations and the calculation of the absolute value of a real number.

It was presented a numerical scheme for carrying out numerical simulations as well as some typical results.

It must be (again) pointed out that the considered methodology has no limit of accuracy and is computationally cheap and easy to be implemented. These advantages make the proposed approach the first choice for simulating problems with concentration-dependent diffusion coefficient.

In fact, the procedure considered in this work is not restricted to Fick's second law. Transient heat transfer phenomena with temperature-dependent thermal diffusivity (and temperature-dependent thermal conductivity) may be simulated by means of the protocol presented here. In addition, any partial differential equation with similar structure is eligible to be numerically solved with the aid of the tools presented here.

As a first hint for future research, we could consider the use of Robin boundary conditions and/or Dirichlet boundary conditions varying in time.

Besides, some transient problems, modelled under the Continuum Mixture Theory viewpoint $[27,28]$, could be simulated with the tools presented in this work.

\section{References:}

[1] Fick, A., On Liquid Diffusion, The London, Edinburgh, and Dublin Philosophical Magazine and Journal Science, Vol. 10, 1855, pp. 30-39.

[2] Han, C. C. and Akcasu, A. Z., Concentration dependence of Diffusion Coefficient at various Molecular weights and Temperatures, Polymer, Vol. 22, 1981, pp. 1165-1168.

[3] Stokes, R. H., One-dimensional Diffusion with the Diffusion Coefficient a Linear Function of Concentration, Transactions of the Faraday Society, Vol. 48, 1952, pp.887-892.

[4] Gaede, H. C., Gawrisch, K., Lateral Diffusion Rates of Lipid, water and a Hydrophobic Drug in a Multilamellar Liposome, Biophys. J., Vol. 85, 2003, pp. 1734-1740.
[5] Incropera, F. P., Dewitt, D. P., Bergman, T. L. and Lavigne, A, S., Fundamentals of Heat and Mass Transfer, $6^{\text {th }}$ Edition, John Wiley \& Sons, 2007.

[6] Slattery, J. C., Momentum, Energy and Mass Transfer in Continua, McGraw-Hill Kogakusha 1972.

[7] Botar, L. and Ruff, I., On the General and Special form of Fick's Second Law with Concentration-Dependent Diffusion Coefficient Chemical Physics Letters, Vol. 149, no. 1, 1988, pp.99-101.

[8] Jou, D., Casas-Vazquez, J., Madureira, J. R., Vasconcellos, A. R. and Luzzi, R., High-Order Hydrodynamics: extended Fick's Law, Evolution Equation and Bobylev's Instability, J. Chem. Phys., Vol. 116, no. 4, 2002, pp. 1571-1584.

[9] Van Milligen, B. P., Bons, P. D., Carreras, B. A. And Sanchez, R., On the Applicability of Fick's Law to Diffusion in Inhomogeneous Systems, Eur. J. Phys., Vol. 26, no. 5, 2005, pp. 913-925.

[10] Sasaki, T., Gunji, Y. and Iida, T., Transient Diffusion Measurements of Radon Fick's Law Confirmation, J. Nuclear Science Technol., Vol. 44, no. 10, 2007, pp. 1330-1336.

[11] Zhang, J., Wang, J. and Kong, D., Chloride Diffusivity Analysis of Existing Concrete Based on Fick's Second Law, J. Wuhan Technol. Mater. Sci. Ed., Vol. 25, no. 1, 2010, pp. 142-146.

[12] Siepmann, J. and Siepmann, F., Modeling of Diffusion Controlled Drug Delivery, $J$ Control Release, Vol. 161, no. 2, 2011, pp. 351-362.

[13] Guenneau, S. and Puvirajesinghe, T. M., Fick's Second Law Transformed: One Path to Cloaking in Mass Diffusion, $J \quad R$. Soc. Interface, Vol. 10, no. 83, 2013, pp. 1-6.

[14] John, F., Partial Differential Equations, $4^{\text {th }}$ Edition, Springer, 1981.

[15] Wylie, C. R., Advanced Engineering Mathematics, $4^{\text {th }}$ Edition, McGraw-Hill Kogakusha, 1975.

[16] Arpaci, V. S., Conduction Heat Transfer, Addison Wesley Publishing Company, 1966.

[17] Carslaw, H. S. and Jaegger, J. C., Conduction Heat in Solids. Oxford Press, 1959.

[18] Gama, R. M. S., Corrêa, E. D. and MartinsCosta, M. L., An upper bound estimate for the steady-state temperature for a class of heat conduction problems wherein the thermal conductivity is temperature dependent International Journal of Engineering Science, Vol. 69, 2013, pp. 77-83. 
[19] Givoli, D., Use of the Kirchhoff transformation in Finite Element Analysis. International Journal of Numerical Methods for Heat \& Fluid Flow, Vol. 3, 1993, pp. 463-477.

[20] Nilsson, O., Mehling, H., Horn, R. Fricke, J. and Hofmann, D., Determination of the Thermal Diffusivity of Monocrystalline Silicon Carbide, High Temperatures-High Pressures, Vol.29, 1997, pp. 73-79.

[21] Jaber, S., Alahmer, A., Borowski, G. and Alsaqoor, S., Optimal Mass Diffusion Transfer in Solids Using Heat Transfer Similarities, Advances in Science and Technology Research Journal, Vol. 13, no. 4, 2019, pp.54-59.

[22] Afrin, N. Feng, Z. C., Zhang, Y. and Chen, J. $\mathrm{K}$., Inverse estimation of front surface temperature of a locally heated plate with temperature-dependent conductivity via Kirchhoff transformation. Int. J. Thermal Sciences, Vol. 69, 2013, pp. 53-60.

[23] Joyce, W., Thermal Resistance of Heat Sinks with Temperature-Dependent Conductivity," Solid-State Electronics, Vol. 18, 1975, pp. 321-322.

[24] Kim, S., A simple direct estimation of temperature-dependent thermal conductivity with Kirchhoff transformation, Int. Commun. Heat Mass Transfer, Vol. 28, 2001, pp. 537544.

[25] Tomatis, D., Heat Conduction in Nuclear Fuel by the Kirchhoff Transformation, Annals Nuclear Energy, Vol. 57, 2013, pp. 100-105.

[26] Mierzwiczak, M. and Kolodziej, J. A., The Determination of Temperature-Dependent Thermal Conductivity as Inverse Steady Heat Conduction Problem. Int. J. Heat Mass Transfer, Vol. 54, 2011, pp. 790-796.

[27] Gama, R. M. S., Costa, M. L. M. and Sampaio, R., On the Energy Balance for Continuous Mixtures, Mech. Research Communications, Vol. 20, no. 1, 1993, pp. 53-58.

[28] Saldanha da Gama, R. M., A Variational Principle for the Steady-State Heat Transfer Process in a Rigid Continuous Mixture, Math. Problems in Engineering, Vol. 21, 2021, pp. 110.

\section{Author Contributions:}

R. M. S. Gama proposed the general piecewise approximation, carried out the error estimate and wrote the FORTRAN code.

R. Pazetto S. da Gama proposed the numerical approach, carried out the numerical simulations and created the code for drawing the figures.

\section{Sources of funding:}

The author R. M. S. Gama gratefully acknowledge the financial support provided by the brazilian agency CNPq (Grant: 306364/2018-2).

\section{Creative Commons Attribution}

\section{License 4.0 (Attribution 4.0 International , CC BY 4.0)}

This article is published under the terms of the Creative Commons Attribution License 4.0 https://creativecommons.org/licenses/by/4.0/deed.en US 